$\mathrm{U}$ na de las familias de Angiospermas más características de las zonas tropicales de la Tierra es la familia Moraceae, fundamentalmente por su distribución pantropical, su diversificación genérica y específica (40-75 géneros y 10001850 especies), su importancia en la estructura de las comunidades (riqueza de taxa, abundancia y biomasa) y su indudable relevancia económica, particularmente de especies pertenecientes a los géneros Artocarpus Forster \& Forster, Brosimum Sw., Castilla Sessé, Ficus L. y Maclura Nutt. (Berg, 1973; Cronquist, 1981; Heywood, 1985; Gentry, 1988; Mabberley, 1993). Corner (1962) divide en seis tribus a la familia (géneros y especies entre paréntesis): Artocarpeae (15/95), Brosimeae $(8 / 44)$, Dorstenieae $(1 / 120)$, Ficeae $(1 / 1000$ ?), Moreae $(10 / 68)$ y Olmedieae $(18 / 69)$.

El género Ficus es el único representante de la tribu Ficeae y las evaluaciones realizadas con respecto a su diversidad específica sugieren al menos 750 taxa, con una distribución pantropical y subtropical (Berg, 1973, 1989; Corner, 1962; Wiebes, 1986b; Compton et al. 1996). Este género es uno de los taxa más notables, no sólo dentro de Moraceae, sino para las Angiospermas en general, ya que es claramente distinto por su inflorescencia característica casi totalmente cerrada (el sicono o "higo", el cual sólo tiene una apertura apical denominada ostíolo), que requiere para la polinización de sus flores de un fascinante acoplamiento temporal y espacial con avispas específicas de la familia Agaonidae y que, adicionalmente, es la estructura que funciona como la unidad de dispersión (Ramírez, 1969; Corner, 1985; Wiebes 1986a, b; Verkerke, 1989; Ibarra-Manríquez, 1991; Compton et al. 1996).

El género está representado en los trópicos americanos por dos subgéneros, Urostigma Gasparrini

\title{
Estudio palinológico de Ficus, subGénero Pharmacosycea (Moraceae) de Veracruz, MÉXICO
}

\author{
Guillermo IbarRa-ManríQuez ${ }^{1}$ Y \\ Enrique Martínez-Hernández ${ }^{2}$
}

1 Departamento de Botánica, Instituto de Biología. Universidad Nacional Autónoma de México, Apartado Postal 70-233, 04510, México, D. F., México. Dirección actual: Departamento de Ecología de los Recursos Naturales. Instituto de Ecología. Universidad Nacional Autónoma de México. Antigua Carretera a Pátzcuaro No. 8701 Col. San José de la Huerta, Morelia, Michoacán, 58190 México.

2 Departamento de Geología, Instituto de Geología, Universidad Nacional Autónoma de México. Apartado Postal 70-296, 04510, México, D. F. México.

(100-120 especies) y Pharmacosycea Miquel (20-30 especies), ambos presentes en México, los cuales son claramente distintivos en diversos aspectos de su morfología y ecología. En particular, las especies del subgénero Urostigma son epífitas, rupícolas o "estranguladoras", con las semillas rodeadas por una cubierta viscosa, las cuales germinan generalmente sobre ramas gruesas o directamente en los troncos de árboles, los siconos están en pares, con dos (raramente tres) brácteas basales y las flores estaminadas poseen un solo estambre con anteras indehiscentes. Por el contrario, los miembros del subgénero Pharmacosycea son árboles de crecimiento independiente (excepto F. crassiuscula Warb. que es epífito), con semillas no pegagosas que germinan en el suelo, siconos solitarios, con tres brácteas basales o sólo con una bráctea trilobulada, flores estaminadas con dos estambres (raramente uno o tres) y anteras dehiscentes (De Wolf, 1960, 1965; Ramírez, 1976; Berg y Simonis, 1981; IbarraManríquez, 1991). Adicionalmente, en el subgénero Urostigma las flores son específicamente polinizadas por avispas del género Pegoscapus, mientras que en el subgénero Pharmacosycea este mecanismo es realizado exclusivamente por especies del género Tetrapus (Ramírez, 1969; Wiebes 1986a; Compton et al. 1996; Herre et al. 1996).

Desafortunadamente, no existe hasta el momento un estudio holístico que defina claramente los caracteres taxonómicos a considerar en el género Ficus y que permita una adecuada delimitación de sus especies (p. ej. Standley 1917; De Wolf, 1960, 1965; Corner, 1970, 1985; Burger, 1977; Ramírez, 1977; Berg, 1989). Como parte de un estudio taxonómico del subgénero Pharmacosycea (Ibarra-Manríquez y Wendt, 1992), se realizó el presente estudio con el propósito de indagar la relevancia taxonómica de la morfología del polen (especialmente usando el microscopio electrónico de barrido), para la delimitación de las especies del subgénero Pharmacosycea presentes en Veracruz. Las cinco especies de Ficus incluidas en 
este trabajo representan todos los miembros del subgénero encontrados hasta el momento en México: $F$. insipida Willdenow, F. lapathifolia (Liebmann) Miquel, $F$. maxima P. Miller, $F$. petenensis Lundell y $F$. yoponensis Desvaux.

La primera referencia al polen de Moraceae proviene del trabajo pionero de Erdtman (1952), quien la caracterizó como una familia relativamente uniforme (estenopalinológica), con granos bilaterales o radiosimétricos, 2-4 (-5) porados y con forma oblata a prolata. Datos más recientes para especies particu-

Cuadro 1. Ejemplares de referencia y características del polen para las especies de Ficus, subgénero Pharmacosycea presentes en Veracruz. Abreviaturas: $\mathrm{P}=$ eje polar; $\mathrm{E}=$ eje ecuatorial.

\begin{tabular}{l}
$\begin{array}{l}\text { Ejemplares de } \\
\text { referencia }\end{array}$ \\
\hline
\end{tabular}

\begin{tabular}{|c|c|c|c|c|}
\hline F. insipida & & & & \\
\hline Ibarra 3256 & 8.1 & 12.6 & 0.65 & Oblato \\
\hline Beaman 5175 & 8.7 & 13.2 & 0.66 & Oblato \\
\hline Boege 3187 & 10.2 & 15.0 & 0.68 & Oblato \\
\hline Castillo 1610 & 8.5 & 12.4 & 0.68 & Oblato \\
\hline Ibarra 3259 & 9.5 & 13.4 & 0.70 & Oblato \\
\hline F. lapathifolia & & & & \\
\hline Ibarra 3218 & 8.9 & 14.2 & 0.63 & Oblato \\
\hline Ibarra 3266 & 9.1 & 14.0 & 0.65 & Oblato \\
\hline Ibarra 3267 & 9.6 & 14.2 & 0.68 & Oblato \\
\hline $\begin{array}{l}\text { Brigada Vaz- } \\
\text { quez } 1646\end{array}$ & 10.0 & 14.5 & 0.69 & Oblato \\
\hline Avendaño 749 & 12.2 & 16.1 & 0.76 & Suboblato \\
\hline F. maxima & & & & \\
\hline Ibarra 3241 & 8.7 & 13.1 & 0.66 & Oblato \\
\hline Ibarra 3212 & 9.4 & 13.2 & 0.71 & Oblato \\
\hline Castillo 2332 & 11.1 & 15.4 & 0.72 & Oblato \\
\hline Ibarra 3144 & 10.5 & 14.4 & 0.73 & Oblato \\
\hline Robles 216 & 11.2 & 14.1 & 0.79 & Suboblato \\
\hline F. petenensis & & & & \\
\hline Ibarra 3253 & 8.8 & 11.5 & 0.76 & Suboblato \\
\hline Ibarra 3250 & 9.1 & 11.7 & 0.78 & Suboblato \\
\hline Ibarra 640 & 9.0 & 11.3 & 0.80 & Suboblato \\
\hline Ibarra 3255 & 8.3 & 10.3 & 0.80 & Suboblato \\
\hline Wendt 2654 & 9.5 & 11.7 & 0.80 & Suboblato \\
\hline F. yoponensis & & & & \\
\hline Ibarra 3285 & 8.3 & 12.1 & 0.68 & Oblato \\
\hline Ibarra 3252 & 9.2 & 13.1 & 0.70 & Oblato \\
\hline Cedillo 3228 & 10.1 & 14.3 & 0.71 & Oblato \\
\hline Ibarra 3221 & 9.0 & 12.3 & 0.73 & Oblato \\
\hline Ibarra 3237 & 9.9 & 12.6 & 0.78 & Suboblato \\
\hline
\end{tabular}

lares pueden ser encontrados Melhem (1966), Heusser (1971), Hamilton (1976), Niezgoda y Nowaczyk (1976), Martínez-Hernández et al. (1979), Bonnefille y Riollet (1980), Punt y Eetgerink (1982), Verkerke (1986), Barth (1984) y Hoen y Punt (1989). Para las especies mexicanas existen dos importantes contribuciones: Palacios-Chávez (1967) y Ramos-Zamora (1977). Ambos trabajos coinciden en describir los granos de polen de Ficus como oblatos, muy pequeños $(10-25 \mu)$, la proporción del eje polar y ecuatorial entre $0.43-0.78$, con superficie psilada, 2-3 porado, el poro con un diámetro de $1-2 \mu$, columelas no evidentes, con la exina de (0.8-) $1-1.6 \mu$ y la ectexina y la endexina de similar grosor.

Hasta donde sabemos, existen sólo dos referencias al estudio del polen de la familia con microscopio electrónico de barrido. La primera de éstas es la publicación de Barth (1984), quien señala particularmente que el polen de Ficus es aparentemente psilado bajo el microscopio de luz, mientras que observando la superficie de la exina al electrónico de barrido, es posible ubicarlo como un patrón morfológico distinto de los encontrados en otros 18 géneros de la familia, ya que Ficus tiene una superficie ligeramente irregular, producida por microperforaciones continuas en el tectum, sin espínulas o escabras. La segunda referencia mencionada está restringida a la tribu Dorstenieae (Hoen y Punt, 1989), en donde los autores encontraron información palinológica valiosa para la circunscripción de los miembros de Dorstenieae, particularmente del diverso género Dorstenia (105 especies).

Barth (1984) sugiere también estudiar la estructura de la exina con el microscopio de transmisión, tal y como lo hicieron Martínez-Hernández et al. (1979), con el propósito de lograr una mejor comprensión de las relaciones evolutivas dentro de los miembros de Moraceae. Finalmente, Ramos-Zamora (1985) sugiere las probables tendencias evolutivas dentro del orden Urticales (al cual pertenece Moraceae), con base en las características de los granos de polen, ubicando a Ulmaceae como la familia con el tipo más primitivo de polen, mientras que los de Moraceae y Urticaceae los considera como los más avanzados.

Se estudiaron diversas poblaciones de las cinco especies de Ficus, subgénero Pharmacosycea, en el estado de Veracruz, entidad localizada entre $17^{\circ} 10^{\prime} / 22^{\circ} 29^{\prime}$ N y $93^{\circ} 36^{\prime} / 98^{\circ} 37^{\prime} \mathrm{O}$, México. El material polínico fue obtenido de siconos maduros de diferentes ejemplares depositados en el Herbario Nacional (MEXU), perteneciente al Instituto de Biología de la Universidad Nacional Autónoma de México (UNAM) (cuadro 1). Datos precisos sobre las localidades de cada ejemplar de referencia pueden ser consultados en IbarraManríquez y Wendt (1992). La observación de los 
Polen de Ficus (Moraceae) de Veracruz, México

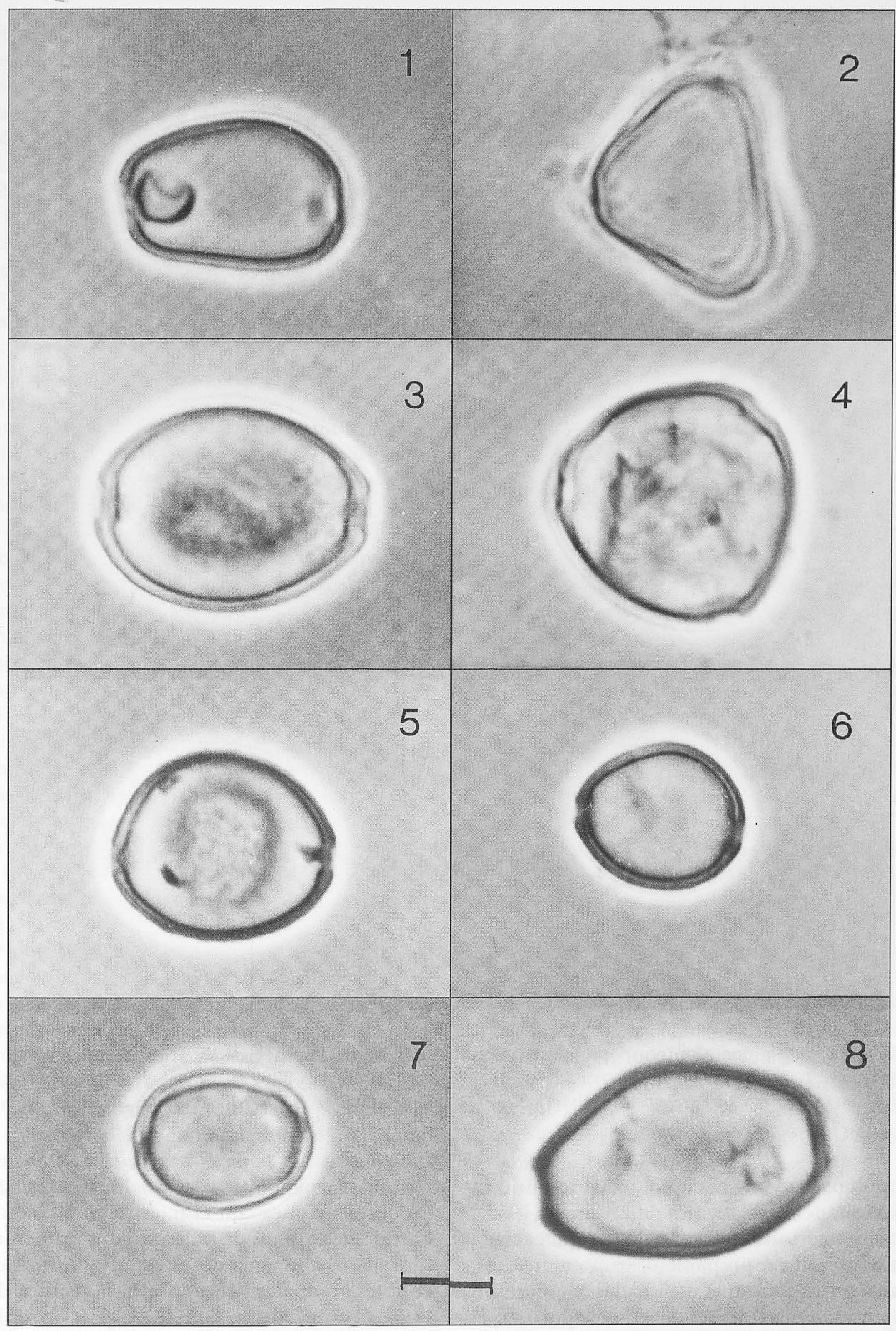

Figura 1. Granos de polen con el microscopio de luz de las especies de Ficus, subgénero Pharmacosycea de Veracruz. 1, 2] Ficus insipida (Ibarra 3256), 3, 4] F. lapathifolia (Ibarra 3267), 5] F. maxima (Ibarra 3212), 6, 7] F. petenensis (Ibarra 3250) y 8] F. yoponensis (Ibarra 3237). Escala $=5 \mu$. 


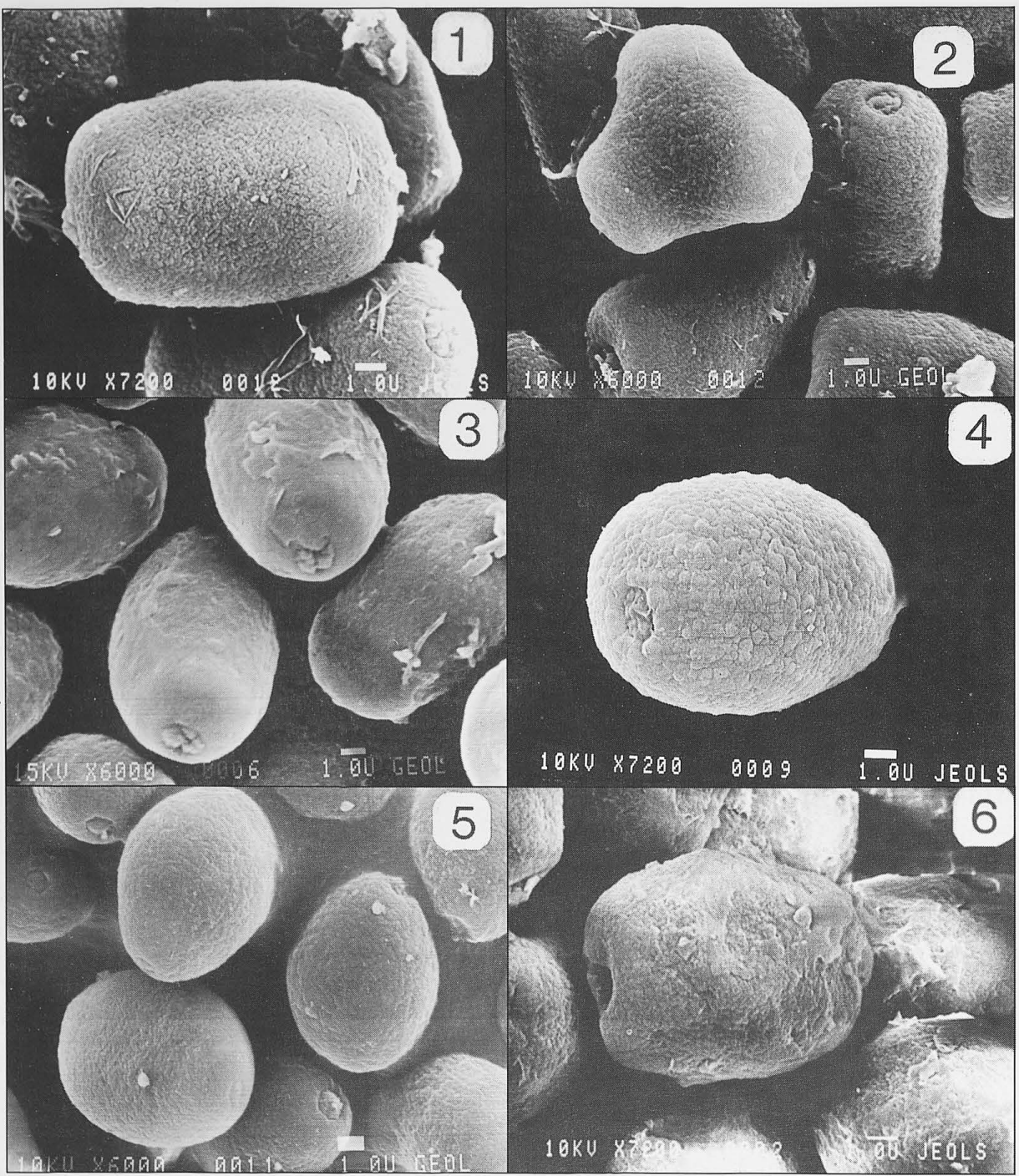

Figura 2. Granos de polen al microscopio electrónico de barrido de las especies de Ficus, subgénero Pharmacosycea de Veracruz. 1, 2] Ficus insipida (Ibarra 3259), 3] F. lapathifolia (Ibarra 3267), 4] F. maxima (Ibarra 3212), 5] F. petenensis (Ibarra 3255) y 6 ] F. yoponensis (Ibarra 3237). Ornamentación: 1] microrrugulada; 2-6] microverrugada.

granos de polen en el microscopio de luz requirió su acetolización siguiendo la técnica de Erdtman (1952), montándose posteriormente en gelatina glicerinada para la elaboración de preparaciones permanentes. En cada una de las muestras estudiadas se midieron 25 granos en vista ecuatorial con el mesoporo en el centro, usando un microscopio Zeiss Kpl, con un aumento de $1250 x$. Se tomaron fotografías en un microscopio Zeiss 55676. Las preparaciones de polen se encuentran depositadas en las colecciones del Ins- tituto de Geología (UNAM) y del Herbario MEXU. Para las observaciones con el microscopio electrónico de barrido, los granos de polen fueron primeramente rehidratados con agua destilada, deshidratados con alcoholes graduales y secados por la técnica de "punto crítico", cubriéndose con una capa de oro. Estas preparaciones fueron observadas en el microscopio JEOL del Colegio de Postgraduados, Chapingo y en el microscopio JSM-35C del Instituto de Geología, usando para la fotografía una película Kodak Verichrome 
pan VP120 blanco y negro. y se describieron de acuerdo a la terminología de Erdtman (1952) y Punt et al. 1994.

Los granos de polen de las especies de Ficus, subgénero Pharmacosycea, se observan al microscopio de luz como oblatos o suboblatos, diporados o triporados, con el tectum psilado, pequeños, con el eje polar $(\mathrm{P})$ de $8.1-12.2 \mu$, eje ecuatorial (E) de $10.3-16.1 \mu$ y un cociente $\mathrm{P} / \mathrm{E}$ de $0.63-0.80$ (cuadro 1, lámina 1 ). Los granos de polen triporados fueron encontrados solamente en $F$. insipida y $F$. lapathifolia. El diámetro de los poros oscila entre $0.8-2 \mu$, con los más pequeños presentes en $F$. maxima $(0.8-1 \mu)$ mientras que los de diámeros mayores se encontraron en $F$. insipida y $F$. lapathifolia $(1-2 \mu)$. Es importante constatar que las dimensiones de los ejes de los granos de polen varían a nivel individual, encontrándose incluso valores similares entre individuos pertenecientes a diferentes especies (cuadro 1). En términos generales, $F$. petenensis es la especie con el polen más característico del género, ya que siempre tiene granos suboblatos (raramente presentes en otras especies), con el eje ecuatorial de 10.3-11.7 4 , lo cual lo separa claramente del resto de sus congéneres en Veracruz.

La ornamentación del grano de polen observada al microscopio electrónico de barrido, no permite la separación entre las diferentes especies del subgénero Pharmacosycea (figura 2), y es muy similar a los granos de polen de las dos especies de Ficus estudiadas por Barth (1984). La ornamentación mostró una alta variabilidad entre las distintas colecciones y dentro del material de un mismo individuo, con granos con ornamentación microverrugada a más o menos microrrugulada. En resumen, la única especie con granos de polen distintivos es $F$. petenensis, ya que en general la morfología polínica de las especies del subgénero en Veracruz es muy similar.

Tomando en cuenta los diversos antecedentes polínicos para el género y los resultados encontrados en el presente estudio, parece existir una alta congruencia de la morfometría de su polen (p. ej. ornamentación, forma o tamaño). Los resultados del presente estudio apoyan la utilidad del polen de $\mathrm{Fi}$ cus en la circunscripción genérica dentro de la familia Moraceae y su relevancia marginal en la sistemática de las especies del subgénero Pharmacosycea de Veracruz.

\section{Agradecimientos}

Este trabajo formó parte de la tesis de maestría del primer autor realizada en el Centro de Botánica del Colegio de Postgraduados, Chapingo, Estado de México, para la cual obtuvo una beca del Consejo Nacional de Ciencia y Tecnología (CONACyT), Méxi- co. Agradecemos la asistencia técnica en el microscopio electrónico de barrido de Jorge Váldez (Colegio de Postgraduados, Chapingo) y Margarita Reyes Salas (Instituto de Geología, UNAM). La revisión crítica de María de Lourdes Rico Arce, Ivón Mercedes Ramírez Morillo y Fernando Chiang Cabrera permitió mejorar la estructura e información del trabajo. Finalmente, el Sr. Felipe Villegas colaboró amablemente en la preparación de las figuras para la publicación.

\section{Literatura citada}

Barth O.M. 1984. Surface morphology of Brazilian Moraceae Pollen Grains. Boletin del Instiuto Geociencias Universidad Sao Paulo 15:142-149.

Berg C.C. 1973. Some remarks on the classification and differentiation of Moraceae. Mededeelingen von Het Botanisch Museum Herbarium Utrecht No. 386:1-10.

Berg C.C. 1989. Classification and distribution of Ficus. Experientia 45:605-611.

Berg C.C. y Simonis J.E. 1981. The Ficus flora of Venezuela: five species complexes discussed and two new species described. Ernstia 6:1-10.

Bonnefille R. et Riollet G. 1980. Pollens des Savanes d'Afrique Orientale. Centre National de la Recherche Scientifique. Paris.

Burger W.C. 1977. Moraceae. Flora Costaricensis. Fieldiana Botany 40:94-215.

Compton S.G., Wiebes J.T. y Berg C.C. 1996. The biology of fig trees and their associated animals. Journal of Biogeography 23:405-407.

Corner E.J.H. 1962. The classification of Moraceae. Gardens Bulletin Straits Settlements (Singapore) 19:187-252.

Corner E.J.H. 1970. Ficus, subgénero Pharmacosycea with reference to the species of New Caledonia. Philosophical Transations of the Royal Society. London. B. 259:383-433.

Corner E.J.H. 1985. Essays on Ficus. Allertonia 4:125-154.

Cronquist A. 1981. An integrated system of classification of Flowering Plants. Columbia Univ. Press. New York.

De Wolf G.P. Jr. 1960. Ficus (Tourn.) L. Flora of Panama (Moraceae). Annals of the Missouri Botanical Garden 47:146-165.

De Wolf G.P. Jr. 1965. Ficus, subgenus Pharmacosycea in America. Elliotia 4:1-20.

Erdtman G. 1952. Pollen morphology and plant taxonomy in Angiosperms. Chronica Botanica Co., Walthman, Mass.

Gentry A.H. 1988. Changes in plant community diversity and floristic composition on enviromental and geographical gradients. Annals of the Missouri Botanical Garden 75:1-34.

Hamilton A.C. 1976. Identification of East African Urticales pollen. Pollen et Spores 18:22-66.

Herre E.A., Machado C.A., Bermingham E., Nason J.D., Windsor D.M., McCafferty S.S., van Houten W. y Bach- 
mann, K. 1996. Molecular phylogenies of figs and their pollinator wasp. Journal of Biogeography 23:521-530.

Heusser C.J. 1971. Pollen and spores of Chile. University Arizona Press. Arizona.

Heywood V.H. 1985. Las plantas con flores. Reverté. Barcelona.

Hoen P.P. y Punt W. 1989. Pollen morphology of the tribe Dorstenieae (Moraceae). Review of Paleobotany and Palynology 57:187-220.

Ibarra-Manríquez G. 1991. Ficus (Moraceae): un género interesante para estudios en ecología y sistemática tropical. Ciencia 42:283-293.

Ibarra-Manríquez G. y Wendt, T.L. 1992. Taxonomía de Ficus, subgénero Pharmacosycea (Moraceae) en Veracruz, México. Boletín de la Sociedad Botánica de México 52:3-29.

Mabberley D.J. 1993. The plant-book. A portable dictionary of the higher plants. Cambridge Univ. Press, Cambridge.

Martínez-Hernández E., Vargas-Olvera E.M., Ludlow-Wiechers B. 1979. Ultraestructura de la exina en granos de polen de Angiospermas. Biótica 4:33-48.

Melhem T.S. 1966. Pollen grains of plants of the "Cerrado». XII -Cucurbitaceae, Menispermaceae y MoraceaeAnais da Academia Brasileira da Ciencias 38:195-203.

Niezgoda C J. y Nowaczyk J. Jr. 1976. Palynological studies in Acanthinophyllum, Clarisia, Sorocea and Trophis (Moraceae). Pollen et Spores 18:513-522.

Palacios-Chávez R. 1967. Morfología de los granos de polen de árboles del estado de Morelos. Anales de la Escuela Nacional de Ciencias Biológicas de México 16:41-169.

Punt W. y Eetgerink E. 1982. On the pollen morphology of some genera of the tribe Moreae (Moraceae). Grana 21:15-19.

Punt W., Blackmore S., Nilsson S. y Thomas A.L. 1994. Glossary of pollen and spore terminology. LPP Contributions series No. 1. LPP Foundation, Utrech.

Ramírez W.B. 1969. Fig wasp: mechanism of pollen transfer. Science 163:580-581.

Ramírez W.B. 1976. Germination on seed of the New World Urostigma (Ficus) and Morus rubra L. (Moraceae). Revista de Biología Tropical 24:1-6.

Ramírez W B. 1977. A new classification of Ficus. Annals of the Missouri Botanical Garden 64:296-310.

Ramos-Zamora D. 1977. La morfología de los granos de polen de la familia Moraceae en México. Boletín de la Sociedad Botánica de México 36:71-92.

Ramos-Zamora D. 1985. Estudio polínico de algunos géneros mexicanos de Ulmaceae y Urticaceae. En Montúfar A. (Ed). Estudios Palinológicos y Paleobotánicos. Instituto Nacional de Antropología e Historia-Secretaría de Educación Pública. México. 39-65.

Standley P.C. 1917. The Mexican and Central American species of Ficus. Contribution from the United States National Herbarium 20:1-35.

Verkerke W. 1986. Anatomy of Ficus ottoniifolia (Moraceae) syconia and its role in the fig-fig wasp symbiosis. Proceding Konninklijke Neederlandse Akademie van Wetenschappen (Amsterdam) C89:443-469.

Verkerke W. 1989. Structure and function of the fig. Experientia 45:612-622.

Wiebes J.T. 1986a. Agaonidae (Hymenoptera, Chalcidoidea) and Ficus (Moraceae): fig wasps and their figs I. Proceding Konninklijke Neederlandse Akademie van Wetenschappen (Amsterdam) C89:335-355.

Wiebes J.T. 1986b. The association of figs and figs-insects. Revue de Zoologie africaine 100:63-71. 